\title{
银纳米线透明导电薄膜的失效机理研究
}

\author{
叶长辉, 顾瑜佳, 王贵欣, 毕丽丽 \\ (浙江工业大学 材料科学与工程学院, 杭州 310014)
}

摘 要: 银纳米线透明导电薄膜材料作为新兴的无铟电极材料, 以其优越的光电性能和力学柔㓞性, 在显示器件、 触控面板、太阳能电池、智能加热和电磁屏蔽等领域崭露头角, 吸引越来越多的来自科研界及产业界的关注。然而, 银纳米线透明导电薄膜在应用中面临着较为严重的稳定性问题, 主要表现为容易被痕量含硫气体腐蚀, 在 $300{ }^{\circ} \mathrm{C}$ 以 上的温度下纳米线出现断裂和球形化等结构失稳现象, 在紫外光照条件下腐蚀及球形化加剧, 在加载电场条件下 出现离子迁移并产生孔洞及断裂现象。本文详细介绍了以上各种失效现象, 分析了失效的微观机制, 介绍了解决各 种失效现象的具体措施。银纳米线透明导电薄膜失效行为的研究，有助于进一步推动该材料的实际应用进程。

关 键 词: 银纳米线; 透明导电薄膜; 失效机理; 电迁移; 硫化; 综述

中图分类号: TQ174 文献标识码: A

\section{Degradation Mechanism of Silver Nanowire Transparent Conductive Films: a Review}

\author{
YE Chang-Hui, GU Yu-Jia, WANG Gui-Xin, BI Li-Li \\ (College of Materials Science and Engineering, Zhejiang University of Technology, Hangzhou 310014, China)
}

\begin{abstract}
Silver nanowire transparent conductive film is one of the new indium-free electrode materials. It has attracted increasing attention from academia and industry due to its superior optoelectronic properties and excellent flexibility. It has been employed in a wide variety of applications in displays, touch panels, solar cells, smart heaters, electromagnetic interference shielding, and so on. However, silver nanowire transparent conductive film has a serious stability issue in service, for example, break or spheroidization above $300{ }^{\circ} \mathrm{C}$ under fulfidation, accelerating the degradation under ultraviolet light, pores formation or even breakdown due to electromigration. In this review, the degradation phenomena is thoroughly introduced, the degradation mechanisms is analyzed, and the remedy strategy of degradation is discussed.
\end{abstract}

Key words: silver nanowires; transparent conductive films; degradation mechanism; electromigration; sulfidation; review

银纳米线 $(\mathrm{AgNW})$ 是近年来广受重视的一种新 材料, 一般为单晶结构, 平均直径在 20 100 nm, 平 均长度在 $20 \sim 200 \mu \mathrm{m}$, 长径比可达 1000 以上。银纳 米线一般由多元醇还原法制备, 随着研究的深入, 银纳米线的直径和长度等已经可以在一定范围内进 行调控, 并且产率也稳步提高。由银纳米线涂布形 成的网络, 对可见光的透过率可以高达 $90 \%$ 以上, 而连续网络可以形成有效的导电通道, 其方块电阻
可以低至 $30 \Omega / \square$ 以下, 并且随着银纳米线直径的减 小, 网络薄膜对于可见光的散射逐渐降低, 其雾度 可以减小至 $0.5 \%$ 以下。银纳米线透明导电薄膜的这 些性能参数, 已经可以与最好的氧化铟锡(ITO)透 明导电薄膜材料相媲美, 因此, 吸引了学术界和产 业界的广泛关注 ${ }^{[1-7]}$ 。因为铟资源短缺以及 ITO 的脆 性问题, 预期在未来的柔性显示及柔性电子等领域 中, 银纳米线透明导电薄膜将逐步取代 ITO 材料, 
成为最主要的柔性透明导电电极材料。

尽管光电性能已经达到了实际应用的要求, 但 是银纳米线透明导电薄膜的稳定性问题始终是困扰 科学界和工业界的难题。银纳米线不仅容易受到大 气环境中的含硫物质所腐蚀, 而且在加热以及加载 电流时都可能导致结构变化, 乃至导电网络失效, 在光照条件下腐蚀及失效过程会进一步加剧。银纳 米线的结构失稳和功能失效机理比较复杂, 概括起 来, 主要分为化学腐蚀、热失效以及电迁移失效等, 如图 1(a d)所示, 实际应用中可能多种因素同时存 在 ${ }^{[8-27]}$ 。因此, 近几年来, 已经有研究人员陆续提出 了一些解决银纳米线透明导电薄膜失效的措施, 主 要是基于在银纳米线透明导电薄膜表面涂覆金属氧 化物、石墨烯、有机物等透明的保护层，隔绝大 气环境中的腐蚀性化学物质, 以及阻止银离子迁移 等 ${ }^{28-31]}$ 。值得指出的是, 采用任何方法提高银纳米 线透明导电薄膜的稳定性，前提条件是不能明显弱 化薄膜的光电性能, 这也是具有挑战性的问题。本 文将针对银纳米线透明导电薄膜的失效现象和失效 机理展开深入分析，并总结应对各种失效现象的有 效方法。

\section{1 银纳米线的失效形式及其原理}

\section{1 银纳米线的表面腐蚀}

银在大气中的腐蚀主要受大气污染物的种类和 含量的影响。通常认为, 在气态的空气污染物中, 二 氧化硫 $\left(\mathrm{SO}_{2}\right)$ 、硫化氢 $\left(\mathrm{H}_{2} \mathrm{~S}\right)$ 、氯化氢 $(\mathrm{HC1})$ 、二氧化 氮 $\left(\mathrm{NO}_{2}\right)$ 、臭氧 $\left(\mathrm{O}_{3}\right)$ 、羰基硫化物 $(\mathrm{OCS})$ 和氨气 $\left(\mathrm{NH}_{3}\right)$ 是最有害的腐蚀剂 ${ }^{[8-11]}$ 。大量研究表明, 在上述气体

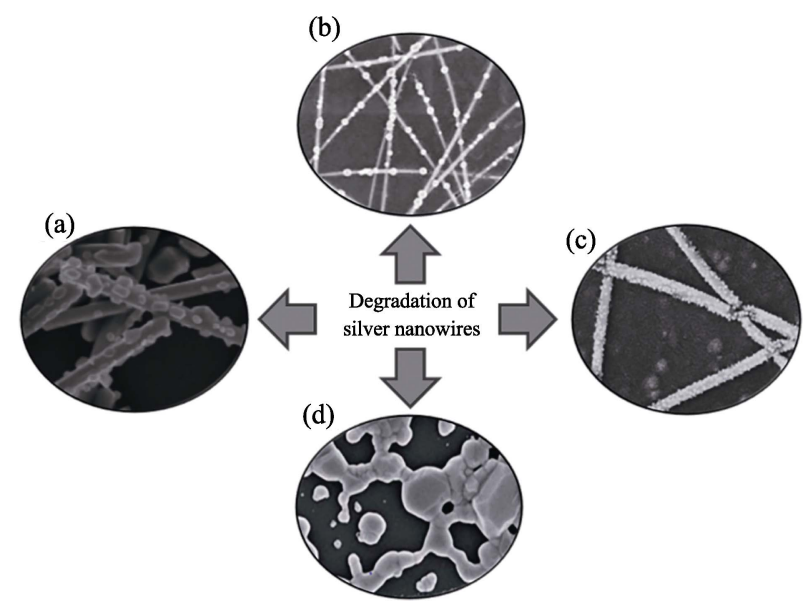

图 1 银纳米线的不同失效形式

Fig. 1 Different failure modes of silver nanowires

(a) Chemical corrosion ${ }^{[10]}$; (b) Thermal failure ${ }^{[15]}$; (c) Joule heat failure $^{[18]} ;$ (d) Electromigration ${ }^{[27]}$
中, $\mathrm{H}_{2} \mathrm{~S}$ 和 OCS 对银的腐蚀最为严重, 尽管他们在 大气中的浓度很低，但在水的配合下，足以在短时 间内使银发生明显的腐蚀 ${ }^{[9]}$ 。与铜和铝等其他金属 相比，银的硫化是更常见的腐蚀方式，而氧化并不 明显, 可能是由于氧化银在常温条件下的热力学稳 定性较低的缘故，因此在本文中不做进一步介绍。

一般认为, 银在受到 $\mathrm{H}_{2} \mathrm{~S}$ 腐蚀之后, 表面会生 成一层硫化银 $\left(\mathrm{Ag}_{2} \mathrm{~S}\right)$ 纳米晶体。其具体反应如方程 式(1)所示 ${ }^{[8]}$ :

$$
2 \mathrm{Ag}+\mathrm{H}_{2} \mathrm{~S} \rightarrow \mathrm{Ag}_{2} \mathrm{~S}+\mathrm{H}_{2}
$$

当有其他气体如氧气 $\left(\mathrm{O}_{2}\right)$ 和 $\mathrm{NO}_{2}$ 存在时, 反应 (1)又会进一步演化成反应方程式(2)和(3) ${ }^{[8]}$ :

$$
\begin{gathered}
2 \mathrm{Ag}+\mathrm{H}_{2} \mathrm{~S}+1 / 2 \mathrm{O}_{2} \rightarrow \mathrm{Ag}_{2} \mathrm{~S}+\mathrm{H}_{2} \mathrm{O} \\
2 \mathrm{Ag}+\mathrm{H}_{2} \mathrm{~S}+2 \mathrm{NO}_{2} \rightarrow \mathrm{Ag}_{2} \mathrm{~S}+2 \mathrm{HNO}_{2}
\end{gathered}
$$

OCS 可以与水反应生成 $\mathrm{H}_{2} \mathrm{~S}$, 从而腐蚀银。其 具体反应如方程式(4)所示 ${ }^{[10]}$ :

$$
\mathrm{OCS}+\mathrm{H}_{2} \mathrm{O} \rightarrow \mathrm{H}_{2} \mathrm{~S}+\mathrm{CO}_{2}
$$

银纳米线在大气环境中的失效形式与体相银相 似。Elechiguerra 等 ${ }^{[10]}$ 对银纳米线在大气环境中的稳 定性做了一系列的研究。图 2 是银纳米线储存于大 气环境中不同时间后拍摄的透射电子显微镜(TEM) 照片。由图中可以看到，刚合成的银纳米线(图 2(a)) 表面是光滑的, 但是在空气中储存三周后变得很粗 糙(图 2(b)), 且周围开始出现纳米粒子。当储存时间 延长至 4、5 和 $24 \mathrm{w}$ 后，可以发现银纳米线表面生 成了一层纳米晶体(图 2(c e ) )。对银纳米线表面纳米 晶的高分辨率 TEM 照片进行快速傅里叶变换(FFT) 分析, 结合图 2(f)和表 1 数据, 可以证实银纳米线表 面的纳米晶体是硫化银。这也说明了虽然大气环境 中的氧气和水汽的浓度比 $\mathrm{H}_{2} \mathrm{~S}$ 高了几个数量级, 但 是硫化银是主要的腐蚀产物。

\section{2 银纳米线的热失效}

银纳米线在均匀的外部温场中, 当温度高于某 一临界温度时会出现瑞利不稳定性现象 ${ }^{[12-15]}$ 。虽然 体相银的熔点在 $960{ }^{\circ} \mathrm{C}$ 以上, 但是由于银纳米线的 比表面积很高, 因此其熔点比体相银要小很多(可 以低至 $300{ }^{\circ} \mathrm{C}$ 左右 ${ }^{[16]}$ 。因为制备银纳米线时一般 采用聚乙烯吡咯烷酮(PVP)作为形貌调节剂，当银 纳米表面残存 PVP 分子时, 纳米线之间的结电阻很 大。在银纳米线透明导电薄膜的制备过程中, 通常 采用退火方法降低银纳米线之间的结电阻，但是众 所周知, 过高的退火温度会导致银纳米线球形化, 从而使银纳米线转化为孤立的银颗粒 ${ }^{[12-13]}$, 这就会 使银纳米线失去渗流特性, 最终导致薄膜功能失效 ${ }^{[17]}$ 。 银纳米线在加热过程中的形态演变过程如图 3(a d) 

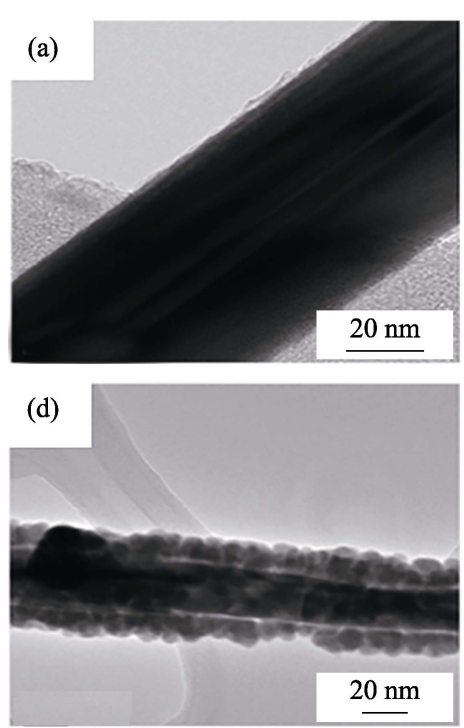

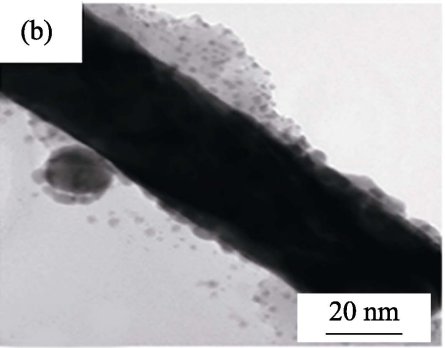

(e)

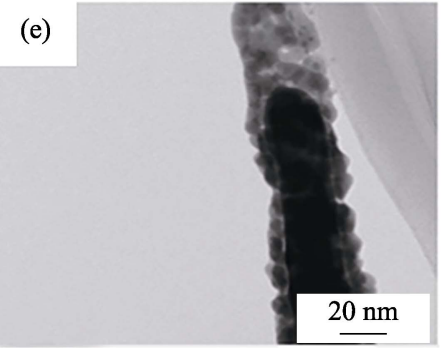

(c)
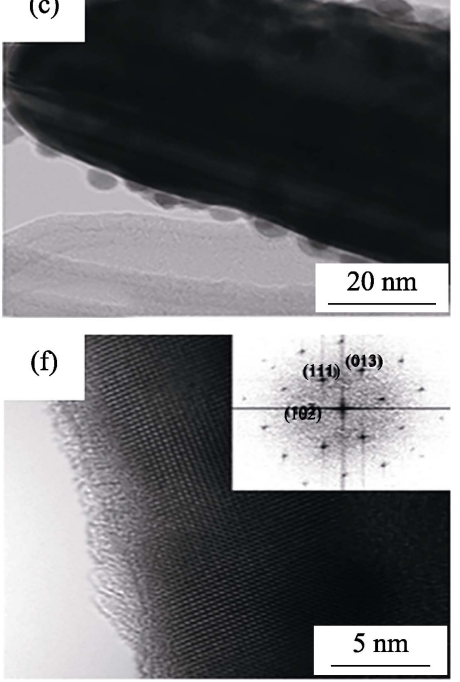

图 2 同一银纳米线样品在环境条件下放置不同时间的透射电镜照片 ${ }^{[10]}$

Fig. 2 TEM images of the same AgNW sample stored for different time after exposure to air at ambient condtions ${ }^{[10]}$ (a) The sample just after synthesis; (b) The sample stored for $3 \mathrm{w}$; (c-e) The sample stored for 4, 5, and $24 \mathrm{w}$, respectively; (f) High-resolution TEM image of one of the crystallites that compose the shell with inset showing to the FFT of the image

表 1 图 2(f)经傅立叶变换(FFT)后测量 所得晶面间距和晶面夹角 ${ }^{[10]}$

Table 1 Interplanar distances and angles between lattice planes from the FFT in Fig. 2(f) ${ }^{[10]}$

\begin{tabular}{ccc}
\hline Parameters & Measured values & Theoretical values \\
\hline$d_{(013)} / \mathrm{nm}$ & 0.242 & 0.242 \\
$d_{(111)} / \mathrm{nm}$ & 0.305 & 0.308 \\
$d_{(10 \overline{2})} / \mathrm{nm}$ & 0.311 & 0.311 \\
$(013) \angle(111) /\left(^{\circ}\right)$ & 49.1 & 50.0 \\
$(111) \angle(10 \overline{2}) /\left(^{\circ}\right)$ & 79.2 & 79.4 \\
$(\overline{102}) \angle(013) /\left(^{\circ}\right)$ & 51.7 & 50.6 \\
\hline
\end{tabular}

所示。图 3(a)为两根相邻银纳米线搭接时的示意图。 当银纳米线在退火时, 总表面能的减少驱动结点处 原子的迁移和纳米线结构重构 ${ }^{[12]}$ 。局部烧结降低了 结电阻, 最终也使整个网络的电阻降低(图 3(b) $)^{[17]}$ 。 随着热负荷的持续, 结点处原子的进一步迁移导致 银纳米线结构失稳, 如图 3(c)所示。图 3(d) 展示了银 纳米线在接近失效极限(完全球形化之前)时的扫描 电子显微镜(SEM)照片, 此时薄膜中的银纳米线仅 残存了微弱的连接。

为了进一步了解银纳米线的热失效机理, Hwang 等 ${ }^{[14]}$ 拍摄了在不同温度下热处理的银纳米线的 SEM 照片, 如图 3(e g) 所示。从未进行热处理前银 纳米线的 SEM 照片(图 3(e))中可以看到, 银纳米线 呈现完整的网络形态。对其进行 $200{ }^{\circ} \mathrm{C}$ 的热处理 $20 \mathrm{~min}$ 后, 银纳米线结点处开始退化(图 3(f))。随着 退火温度进一步升高到 $380{ }^{\circ} \mathrm{C}$, 大部分的银纳米线
已经团聚成球状，SEM 照片中已经看不到完整的银 纳米线网络(图 3(g))。此时, 银纳米线薄膜的电阻非 常大, 而且由于银粒子增加, 光散射也会增强, 从 而造成雾度上升。综上, 银纳米线的热失效主要是 热力学驱动的能量最小化效应导致的结构重构引起 的。因为基材和银纳米线处于同一温场之中, 只要 基材不发生明显的热变形, 对银纳米线的稳定性就 不会有太大的影响。

\section{3 银纳米线的焦耳热失效}

当银纳米线用于太阳能电池、有机发光二极管 (OLED)、透明加热器等器件中时, 在器件运行过程 中电流持续流过透明电极，产生的焦耳热会导致银 纳米线局域温度升高, 该效应较均匀外部加热对银 纳米线结构及导电性的破坏效果更严重 ${ }^{[17]}$ 。

与连续导电薄膜(例如 ITO)中电流流经薄膜的 整个区域不同, 在银纳米线电极中, 电流只流经纳 米线网络导电通道 ${ }^{[18]}$ 。又由于在整个银纳米线网络 中, 相邻两根银纳米线之间的连接处电阻最大 ${ }^{[19-20]}$, 因而在有电流通过时, 结点处的温度便会高于银 纳米线网络其他区域的温度，形成局域化的“热点” (图 4(a)) $)^{[22]}$ 。特别是对于基材导热性较差的器件, 银 纳米线产生的焦耳热难以通过基材向外扩散, 进一 步导致了局域热量的累积。

据 Khaligh 等 ${ }^{[21]}$ 报道, 当银纳米线电极在电流 密度为 $10 \sim 20 \mathrm{~mA} / \mathrm{cm}^{2}$ 的条件下工作时, 两天之内 便会失效。电极失效是由于焦耳加热导致银纳米线 断裂, 从而在银纳米线网络中产生电不连续性, 破 

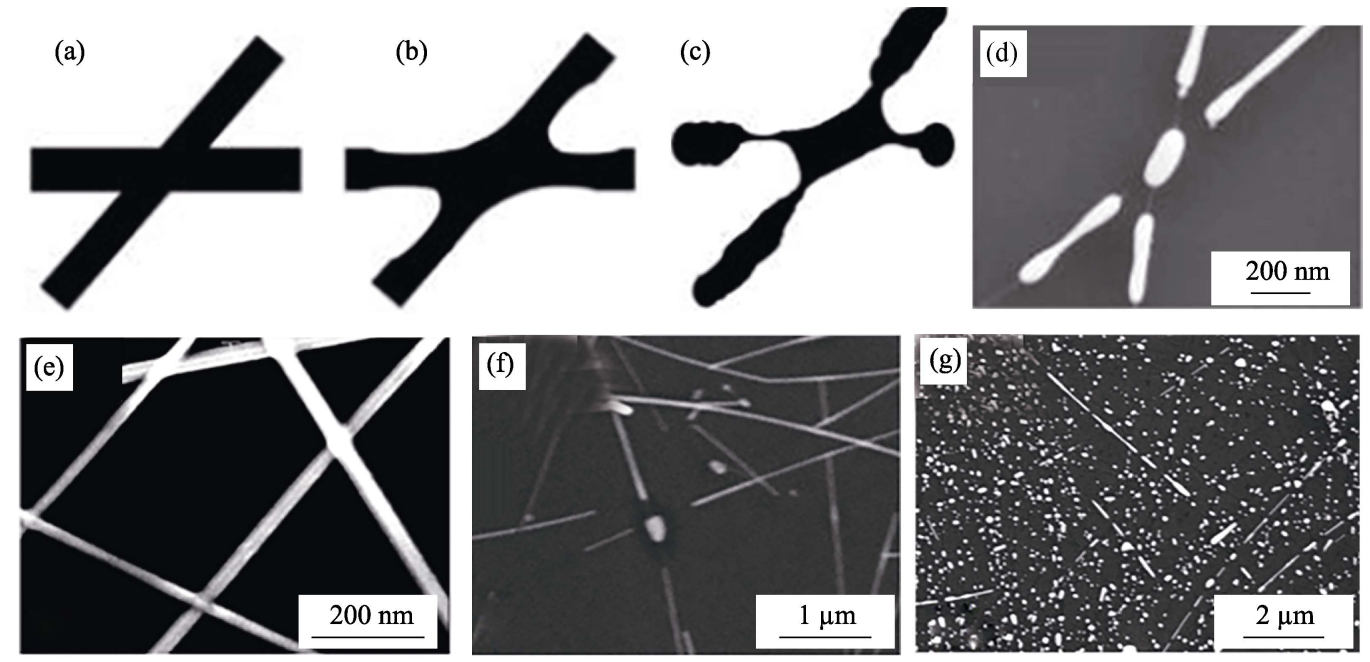

图 3 两根相邻银纳米线之间的连接示意图 ${ }^{[12]}$

Fig. 3 Schematic representation of a junction between two adjacent $\mathrm{AgNW}^{[12]}$

(a) As-deposited junction; (b) Local sintering; (c) Initiation of the deterioration of the junction; (d) SEM image of a AgNW junction after thermal load just before the failure point; (e-g) SEM images of bare AgNW electrodes

(e) Before annealing and after annealing for (f) $200{ }^{\circ} \mathrm{C}, 20 \mathrm{~min}$ and (g) $380{ }^{\circ} \mathrm{C}, 20 \mathrm{~min}^{[14]}$

(a)

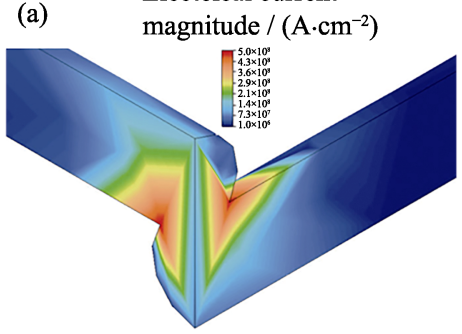

(d)

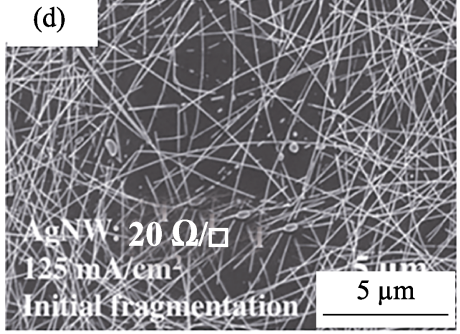

(b)

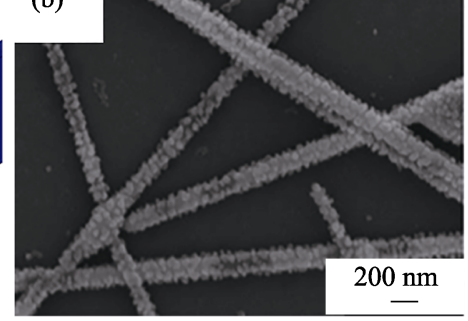

(e)

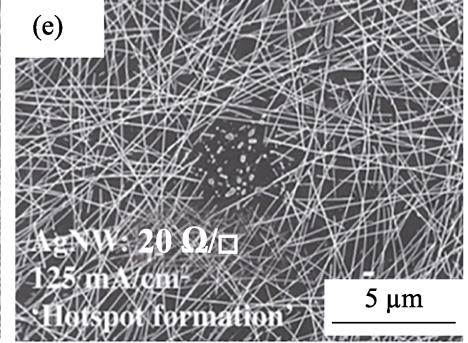

(c)

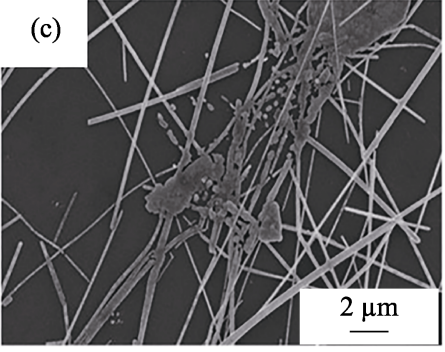

\section{(f)}

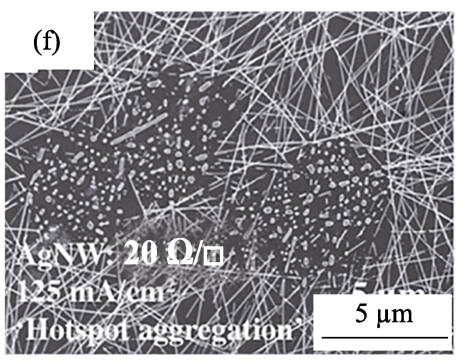

图 4 (a)电流通过两根银纳米线网络结点的有限元模拟 ${ }^{[22]}$; (b,c) 方阻为 $12 \Omega / \square$ 的银纳米线电极持续流过 电流密度为 $17 \mathrm{~mA} / \mathrm{cm}^{2}$ 的电流 $17 \mathrm{~d}$ 后的 SEM 照片 ${ }^{[21]}$; (d f)银纳米线网络在焦耳热作用下的 SEM 照片 ${ }^{[22]}$ :

(d)银纳米线网络局部断裂; (e)热点的扩大; (f)热点合并, 形成电不连续区域

Fig. 4 (a) Finite-element simulation of the current flow through a two AgNW junction ${ }^{[22]}$; (b, c) SEM images of a $12 \Omega / \square$ AgNW electrode under a constant current density of $17 \mathrm{~mA} / \mathrm{cm}^{2}$ for $17 \mathrm{~d}^{[21]}$; (d-f) SEM image of AgNW network under Joule heating ${ }^{[22]}$ : (d) Local fracture of the AgNW network; (e) Expansion of hot spots; (f) Hot spots merge to form an electrically discontinuous region

坏导电通路。电极失效后典型的 SEM 照片如图 4(b c ) 所示。电流长时间流过电极后, 银纳米线表面出现 了纳米颗粒(图 4(b))。在样品的局部区域, 银纳米线 被分解成不连续的片段, 如图 4(c) 所示。

通过银纳米线网络在焦耳热作用下的 SEM 照 片分析 (图 4(d f ) ) 可以详细地了解到银纳米线电 极结构变化的规律。从图 4(d)中可以看到, 首先发 生断裂的是网络比较稀疏区域的银纳米线结点处。 每当有一个结点发生失效, 可用来导电的纳米线
数量就会减少。如果继续对样品施加恒定电流, 结 点周围区域的导电路径将会承受比之前更高的电流 密度，同时，温度也会进一步上升，图 4(e)中的“热 点”将会不断扩大。若电流持续通过，这些不连续的 小片段最终将会合并, 如图 4(f)所示, 致使银纳米 线网络中出现大面积的电不连续区域, 严重破坏电 极的导电通路, 导致焦耳热失效。焦耳热失效通常 表现为“雪崩式”爆发, 往往伴随着基材的严重 变形。 


\section{4 银纳米线的光降解}

银纳米线在各种应用中通常作为透明电极工作, 因此不可避免地会受到紫外光和可见光的照射。与 其他电极材料不同的是, 银纳米线在长期光照条件 下表现出独特而复杂的行为 ${ }^{[23]}$ 。光照会在银纳米线 上产生表面等离激元共振效应, 使得银纳米线网络 局域温度升高, 形成“热点”。与焦耳热效应类似，诱 导银离子的迁移，降低银纳米线之间的结电阻，同 时, 过度的光照也加速了银纳米线向球形纳米颗粒 的转化 ${ }^{[24]}$ 。此外, 除了银颗粒的产生和迁移外, 光 照还会加速银纳米线表面的硫化 ${ }^{[25]}$ 。这些都会影响 银纳米线电极的实际应用。

对银纳米线在光照条件下形貌变化的研究表明 在光照诱导下生成的银纳米颗粒主要沿银纳米线呈 直线分布 (图 5(a)), 生成原因是光照加剧了银纳米 线的硫化 ${ }^{[23]}$ 。大的银纳米线颗粒主要分布于两根银纳 米线的结点处, 形貌呈多面体状而不是球形, 其成 分是 $\mathrm{Ag}$, 主要是由银离子的迁移而产生的(图 5(b d ))。 虽然在一开始这种大颗粒的生成可以降低结点处的 电阻, 但是大纳米颗粒的过度生长也会导致纳米线 直径的损失, 最终造成银纳米线的断裂。归根到底, 银纳米线的光降解本质上也是一种热效应, 与焦耳 热相似，该热效应也会形成局域化的“热点”，从而 显著加速银纳米线的结构失稳过程。

\section{5 银纳米线的电迁移}

银纳米线的电不稳定性可以分为两种状态。除 了上述的焦耳热失效之外, 还有一种失效形式称为 电迁移。电迁移通常是指在电场作用下，金属离子
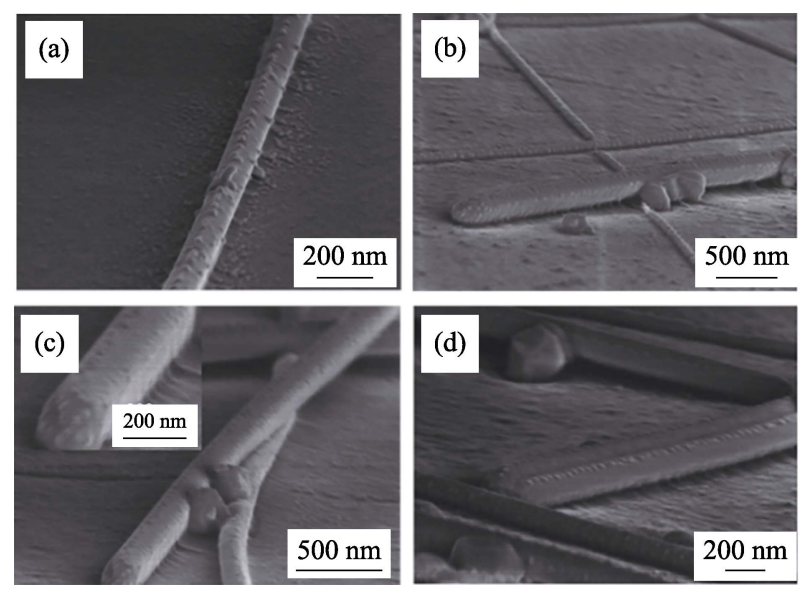

图 5 光照后银纳米线上或周围出现的大、小颗粒的形貌图 ${ }^{[23]}$

Fig. 5 The morphology of the small nanodots and large particles emerged on/around silver nanowires after light irradiation ${ }^{[23]}$ (a) The small nanodots on/around single AgNW; (b) The small nanodots on/around AgNWs with different diameters; (c) The small nanodots at the end of AgNW and also the large particle at the wire-wire junction with inset showing the high magnification image; (d) The large particle adjoined AgNWs
发生迁移的现象。而银的静电放电损伤阈值较低, 因此很容易发生电迁移现象 ${ }^{[20]}$ 。

一般认为, 电迁移作为一种物质的传输形式, 通常是可逆的，其方向不仅与所施加的电场的极性 有关，也与金属材料的晶体结构有关。电迁移可以通 过表面、晶界以及体相进行物质输运。电迁移的微 观驱动力包括电子风和电场力两种。Sindermann 等 ${ }^{[26]}$ 研究发现, 电迁移的过程只是在电子的传输方向上 是可逆的, 但是对于微观结构来说, 电迁移是一种 不可逆的失效形式。即使在电迁移的过程中产生的 孔洞能够被原子填满, 但是整个过程形成了新的晶 界, 对晶格造成了永久性的破坏, 从而缩短了银纳 米线电极的寿命。

通过图 6 可以清楚地看到在电场力作用下空位 的产生及其运动方向。图 6(b i) 表明, 空位运动方向 始终与电流流动方向相反。仔细观察图 6(f)还可以 在空位的运动轨迹中看到一条模糊的黑线。这表明 空位运动会导致晶格退化, 为反向电流下的空位运 动定义了一条首选路径。因此可以看到在不同方向 的电流作用下，初始路径(红线)与反向路径(蓝色)是 重合的, 即电迁移在方向上的可逆。图 $6(\mathrm{k} \sim \mathrm{n})$ 更详 细的描述了银纳米线网络在不同失效阶段的电迁移 现象。从图 6(1 n)可以看到, 在失效过程中, 银颗粒 的尺寸逐渐增大, 电迁移使得较小的银颗粒迁移形 成较大的银颗粒, 此时可以看到出现了较小的空位 团(图 6(1))。这些颗粒将继续生长, 直到彼此分离, 形成更大的孔洞(图 6(m))。电迁移过程会减少有效 的导电通道数目, 导致电流密度增大, 从而进一步 增强电迁移, 最终导致整个银纳米线网络失效。这 主要是由于电流密度越大, 银原子的动量越大, 温 度越高, 电迁移过程越快 ${ }^{[27]}$ 。电迁移失效并不是一 种孤立的过程, 而是一种热激励的过程, 因此, 与 焦耳热失效是紧密相关的。焦耳热效应会加速电迁 移过程，反之则并不一定成立。

\section{2 解决措施}

综合上述分析, 银纳米线的失效机理基本上可 以分为三大类。一是大气中的腐蚀气体对其产生的 腐蚀; 二是银纳米线在均匀外部加热、表面等离激 元共振加热以及局域焦耳热效应下发生的瑞利不稳 定现象, 即断裂及球形化; 三是银纳米线在电场力 作用下发生的电迁移失效。针对这三种失效形式, 具体的解决措施如下。 

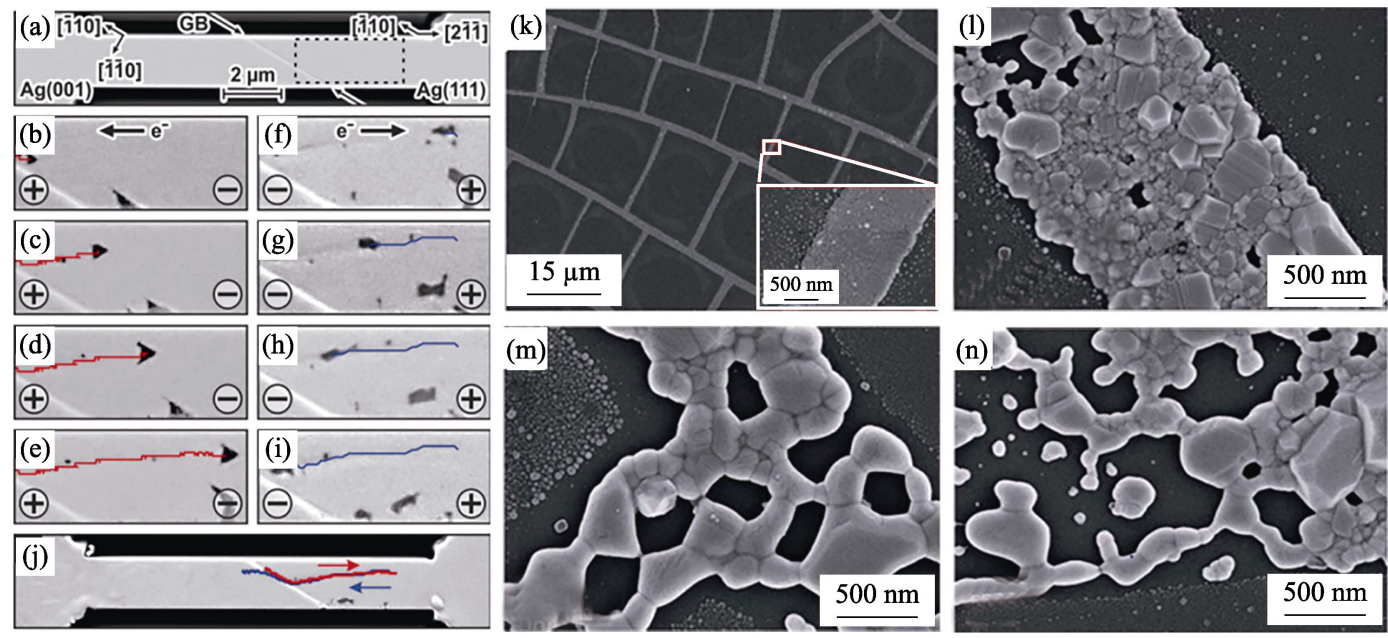

图 6 双晶银纳米线在 $54 \mathrm{~mA}$ 电流下的 SEM 照片 ${ }^{[26]}$

Fig. 6 SEM images of a bi-crystalline AgNW under a current of $54 \mathrm{~mA}^{[26]}$

(a) Prior electrical stressing; (b-e) The direction of vacancy movement when the current flows to the left; (f-i) The direction of vacancy movement when the current moves to the right; (k-n) Surface morphology SEM images of the AgNW network in different stages of degradation ${ }^{[27]}$ :

(k) Fresh sample; (1) Degradation with larger grain size; (m) The emergence of larger voids; (n) Complete breakdown

\section{1 抗腐蚀稳定性}

到目前为止，研究人员为了提高银纳米线电极 在环境条件下的稳定性, 已经开展了大量的研究工 作。为了提高银纳米线抵抗气体腐蚀的能力, 比较 常用的方法是将银纳米线嵌入聚合物基材中, 以隔 绝腐蚀性气体与银纳米线的直接接触。这种措施在 一定程度上减缓了银纳米线的腐蚀, 但是由于聚合 物的厚度必须限制在纳米尺度以保证薄膜的导电性, 所以不能完全阻挡气体的渗透, 仍然难以完全消除 银线的腐蚀现象 ${ }^{[28]}$ 。大面积单层石墨烯对气体的阻 隔效果更好, 也被用于作为封装层来提高银纳米线 的耐腐蚀性, 同时不会明显弱化薄膜的光电性能, 但是缺点是制备方法较复杂 ${ }^{[29]}$ 。最近, Liu 等 ${ }^{[28]}$ 用自 组装的有机硫醇, 2-颈基苯并咪唑(MBI)作为银纳米 线的抑制剂，由于 MBI 抑制剂分子的颈基与银具有 很强的亲和力, 因此可以形成牢固的 $\mathrm{Ag}-\mathrm{S}$ 共价键, 从而形成一层对腐蚀介质 (如 $\mathrm{H}_{2} \mathrm{~S}$ ) 具有阻挡作用的 保护层, 且最终形成的复合电极在高温高湿环境 $\left(85{ }^{\circ} \mathrm{C}\right.$ 和 $85 \mathrm{RH} \%$ )下, 储存 $4 \mathrm{~m}$ 后仍表现出优异的结 构和性能稳定性(图 7(a))。总之, 在银纳米线表面形 成阻碍气体透过的保护层, 是目前比较可行的提高 银纳米线抗腐蚀稳定性的方案。

\section{2 热稳定性}

为了提高银纳米线的热稳定性, 可以采用热稳 定性优异的材料，例如氧化石墨烯 ${ }^{[13]}$ 、还原氧化石 墨烯 ${ }^{[15]} 、 \mathrm{TiO}_{2}{ }^{[30]} 、 \mathrm{ZnO}^{[31]} 、 \mathrm{Al}_{2} \mathrm{O}_{3}{ }^{[14]}$ 等来封装银纳 米线电极, 抑制银原子的扩散, 从而在一定程度上 提高银纳米线的热稳定性。其难点是为了使这种复
合电极能够广泛应用，封装层应该通过低成本、无 真空、可扩展的方法来制备，且应满足其厚度可调 控, 不弱化薄膜的光电性能。目前报道的方法(原子 层沉积、自旋涂层和溅射)取得了较好的效果，同时 也都存在成本高、耗时长、产量低、难度高等缺点。 最近, Khan 等 $^{[12]}$ 提出的大气压力空间原子沉积 (AP-SALD), 是一种可以在大气压力下工作的新的 沉积方法。通过 AP-SALD 制备的 ZnO-AgNW 复合 电极在 $500{ }^{\circ} \mathrm{C}$ 的条件下依旧保持良好的稳定性，而 普通的纯银纳米线电极能承受的温度仅有 $300{ }^{\circ} \mathrm{C}$ (图 7(b))。此外, $\mathrm{ZnO}$ 保护层对银纳米线透明导电薄 膜的光电性能影响很小。因此, 如果该方法能进一 步与卷对卷生产工艺相结合, 将成为提高银纳米线 透明导电薄膜热稳定性的较为理想的方案。

\section{3 电迁移稳定性}

近年来金属材料中电迁移的研究较多, 但是对 于银纳米线的电迁移研究并不深入。解决银纳米线 电迁移稳定性问题，主要通过抑制银原子在银纳米 线表面的扩散来实现, 在银纳米线表面包覆保护层 的方法同样会起到效果。例如，上文提到的 Liu 等 ${ }^{[28]}$ 也对提高银纳米线的电稳定性做出了相关研究。原 始的银纳米线加热器在电压为 $9 \mathrm{~V}$ 的情况下, 在不 到 $30 \mathrm{~s}$ 的时间内温度就能上升至 $110{ }^{\circ} \mathrm{C}$, 且持续加 压 $4 \mathrm{~min}$ 后就会发生失效。而用自组装的有机硫醇 $\mathrm{MBI}$ 作为银纳米线的抑制剂后，电极在接通 $12 \mathrm{~V}$ 的 电压后温度也只上升到了 $130{ }^{\circ} \mathrm{C}$, 且持续加压 $10 \mathrm{~min}$ 后电极都没有发生失效现象。除此之外, Khan 等 ${ }^{[12]}$ 也发现，使用 AP-SALD 方法沉积 ZnO 层后，银纳 
(a)

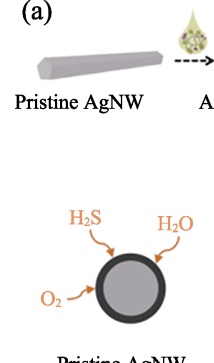

Pristine AgNW

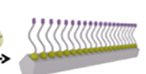

AgNW with SAM

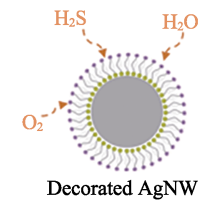

Decorated AgNW

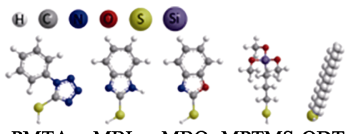

PMTA MBI MBO MPTMS ODT

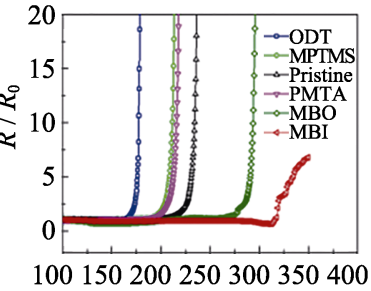

Temperature $/{ }^{\circ} \mathrm{C}$

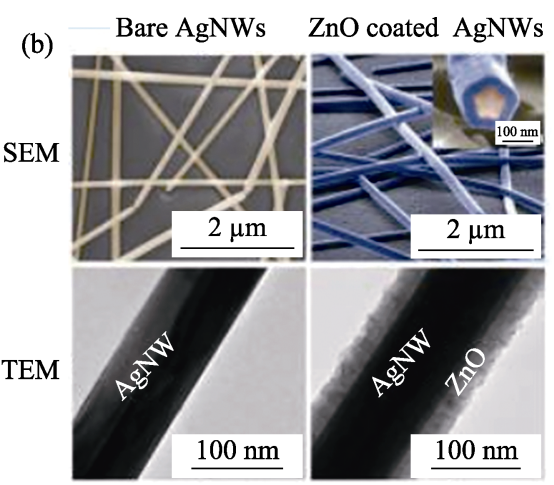

图 7 针对银纳米线的失效形式所提出的具体解决措施

Fig. 7 Remedy strategy of silver nanowire degradation

(a) A self-assembled organic 2-mercaptobenzimidazole (MBI) used as an inhibitor of AgNWs ${ }^{[28]}$;

(b) $\mathrm{ZnO}-\mathrm{AgNW}$ composite electrode prepared by AP-SALD ${ }^{[12]}$

米线的失效电压可以从 $9 \mathrm{~V}$ 提升到最高的 $18 \mathrm{~V}$ 。考 虑到智能手机等的触控屏的驱动电压一般不超过 $12 \mathrm{~V}$, 以上方案如果能在更苛刻的高温高湿条件下 在更长的测试时间内(例如 $200 \mathrm{~h}$ ) 表现出电迁移稳定 性，那么基本上可以达到实际应用的需求。

\section{3 总结与展望}

银纳米线透明导电薄膜材料的结构和功能稳定 性是非常值得关注的重要科学问题，该问题的深入 研究, 是这种战略性新材料最终能够进入市场, 创 造经济和社会价值的前提。本文对于银纳米线及其 透明导电薄膜材料的失效现象进行了总结和归纳, 提出最主要的三种失效现象, 即气体腐蚀、热失稳和 电迁移，并分析了这三种失效现象的微观机制，进 一步提出相应的解决措施。相信, 随着研究的进一步 深入, 银纳米线及其透明导电薄膜材料的失效问题 会得以解决, 而这种重要的新材料也会在柔性电子 等领域发挥其关键作用。

\section{参考文献:}

[1] SHANG Z, LI J, FAN C, et al. In situ study on surface roughening in radiation-resistant Ag nanowires. Nanotechnology, 2018, 29(21): 215708.

[2] BELLET D, LAGRANGE M, SANNICOLO T, et al. Transparent electrodes based on silver nanowire networks: from physical considerations towards device integration. Materials, 2017, 10(6): 570 .

[3] SUN Y, GATES B, MAYERS B, et al. Crystalline silver nanowires by soft solution processing. Nano Lett., 2002, 2(2): 165-168.

[4] LEE J Y, CONNOR S T, CUI Y, et al. Solution-processed metal nanowire mesh transparent electrodes. Nano Lett., 2008, 8(2): 689-692.

[5] LIANG G, YI M, HU H, et al. Coaxial-structured weavable and wearable electroluminescent fibers. Adv. Electron. Mater., 2017, 3(12): 1700401.

[6] SCHUETTE W M, BUHRO W E. Silver chloride as a heterogene- ous nucleant for the growth of silver nanowires. ACS Nano, 2013, 7(5): 3844-3853.

[7] CHEN C, ZHAO Y, WEI W, et al. Fabrication of silver nanowire transparent conductive films with an ultra-low haze and ultra-high uniformity and their application in transparent electronics. J. Mater. Chem. C, 2017, 5(9): 2240-2246.

[8] VOLOE L, PETERSON P J. The atmospheric sulfidation of silver in a tubular corrosion reactor. Corros. Sci., 1989, 29(10): 1179-1196.

[9] FRANEY J P, KAMMLOTT G W, GRAEDEL T E. The corrosion of silver by atmospheric sulfurous gases. Corros. Sci., 1989, 25(2): 113-143.

[10] ELECHIGUERRA J L, LOPEZ L L, LIU C, et al. Corrosion at the nanoscale: the case of silver nanowires and nanoparticles. Chem. Mater., 2005, 17(24): 6042-6052.

[11] GRAEDEL T E, FRANEY J P, GUALTIERI G J, et al. On the mechanism of silver and copper sulfidation by atmospheric $\mathrm{H}_{2} \mathrm{~S}$ and OCS. Corros. Sci., 1985, 25(12): 1163-1180.

[12] KHAN A, NGUYEN V H, ROJAS D M, et al. Stability enhancement of ailver nanowire networks with conformal $\mathrm{ZnO}$ coatings deposited by atmospheric pressure spatial atomic layer deposition. ACS Appl. Mater. Interfaces, 2018, 10(22): 19208-19217.

[13] ZHANG X, YAN X, CHEN J, et al. Large-size graphene microsheets as a protective layer for transparent conductive silver nanowire film heaters. Carbon, 2014, 69: 437-443.

[14] HWANG B, AN Y, LEE H, et al. Highly flexible and transparent $\mathrm{Ag}$ nanowire electrode encapsulated with ultra-thin $\mathrm{Al}_{2} \mathrm{O}_{3}$ : thermal, ambient, and mechanical stabilities. Sci. Rep., 2017, 7: 41336.

[15] AHN Y, JEONG Y, LEE Y. Improved thermal oxidation stability of solution-processable silver nanowire transparent electrode by reduced graphene oxide. ACS Appl. Mater. Interfaces, 2012, 4(12): 6410-6414.

[16] LAGRANGE M, LANGLEY D P, GIUSTI G, et al. Optimization of silver nanowire-based transparent electrodes: effects of density, size and thermal annealing. Nanoscale, 2015, 7(41): 17410-17423.

[17] LAGRANGE M, SANNICOLO T, ROJAS D M, et al. Understanding the mechanisms leading to failure in metallic nanowirebased transparent heaters, and solution for stability enhancement. Nanotechnology, 2017, 28(5): 055709.

[18] KHALIGH H H, XU L, KHOSROPOUR A, et al. The Joule heating problem in silver nanowire transparent electrodes. Nanotechnology, 2017, 28(42): 425703.

[19] SANNICOLO T, CHARVIN N, FLANDIN L, et al. Electrical mapping of silver nanowire networks: a versatile tool for imaging network homogeneity and degradation dynamics during failure. 
ACS Nano, 2018, 12(5): 4648-4659.

[20] FANTANAS D, BRUNTON A, HENLEY S J, et al. Investigation of the mechanism for current induced network failure for spray deposited silver nanowires. Nanotechnology, 2018, 29(46): 465705.

[21] KHALIGH H H, GOLDTHORPE I A. Failure of silver nanowire transparent electrodes under current flow. Nanoscale Res. Lett., 2013, 8(1): 235 .

[22] CHEN D, ZHAO F, TONG K, et al. Mitigation of electrical failure of silver nanowires under current flow and the application for long lifetime organic light-emitting diodes. Adv. Electron. Mater., 2016, 2(8): 1600167.

[23] WANG J, JIU J, ZHANG S, et al. The comprehensive effects of visible light irradiation on silver nanowire transparent electrode. Nanotechnology, 2018, 29(43): 435701.

[24] LEE G P, SHI Y, LAVOIE E, et al. Light-driven transformation processes of anisotropic silver nanoparticles. ACS Nano, 2013, 7(7): 5911-5921.

[25] GRILLET N, MANCHON D, COTTANCIN E, et al. Photo- oxidation of individual silver nanoparticles: a real-time tracking of optical and morphological changes. J. Phys. Chem. C, 2013, 117(5): 2274-2282.
[26] SINDERMANN S P, LATZ A, SPODDIG D, et al. Lattice degradation by moving voids during reversible electromigration. J. Appl. Phys., 2014, 116(3): 034502.

[27] CHEUK K W, PEI K, CHAN P K L. Degradation mechanism of a junction-free transparent silver network electrode. RSC Adv., 2016, 6(77): 73769-73775.

[28] LIU G S, XU Y, KONG Y, et al. Comprehensive stability improvement of silver nanowire networks via self-assembled mercapto inhibitors. ACS Appl. Mater. Interfaces, 2018, 10(43): 37699-37708.

[29] DENG B, HSU P C, CHEN G, et al. Roll-to-roll encapsulation of metal nanowires between graphene and plastic substrate for high-performance flexible transparent electrodes. Nano Lett., 2015, 15(6): 4206-4213.

[30] RAMASAMY P, SEO D M, KIM S H, et al. Effects of $\mathrm{TiO}_{2}$ shells on optical and thermal properties of silver nanowires. J. Mater. Chem., 2012, 22(23): 11651-11657.

[31] CHEN D, LIANG J, LIU C, et al. Thermally stable silver nanowire-polyimide transparent electrode based on atomic layer deposition of zinc oxide on silver nanowires. Adv. Funct. Mater., 2015, 25(48): 7512-7520. 